\title{
Generalisation and Experimental Validation of Design Rules for Self-Phase Modulation-based 2R-Regenerators
}

\author{
L. PROVOST, C. FINOT, K. MUKASA, P. PETROPOULOS, and D. J. RICHARDSON \\ Optoelectronics Research Centre, University of Southampton, Southampton SO17 1BJ, United-Kingdom \\ lap@orc.soton.ac.uk
}

\begin{abstract}
We report the design of 2R-regenerators based on Self-Phase Modulation in lossy fibers. We experimentally demonstrate the applicability of the proposed scaling rules and present detailed characterization of the pulse characteristics at the regenerator output.
\end{abstract}

\section{Introduction}

All-optical signal regeneration techniques relying on the Kerr nonlinearity in optical fibers have proved to be highly suitable for high bit-rate operation. Experimental implementations using Self-Phase Modulation (SPM), Cross-Phase Modulation and Four-Wave Mixing have all been successfully reported. In particular, the technique first reported by Mamyshev [1], which relies on broadening the signal spectrum in a highly nonlinear fiber (HNLF) and filtering by a detuned narrow optical band-pass filter, has attracted much attention over the years. Numerous implementations have been supported either by new fiber structures [2] or by the availability of new highly nonlinear materials (compound glasses [3], bismuth oxide [4]), or conventional HNLFs [5].

Despite the numerous experimental demonstrations in the literature, no generalized design rules linking the physical properties of the SPM-based regenerators to their performance have been reported which would allow a comparison and systematic optimization of such systems. This is mainly because the design of such regenerators is not straightforward and is dependant on a large range of parameters associated with the nonlinear fiber (chromatic dispersion, nonlinearity coefficient and attenuation losses), the filter parameters and central wavelength, the target bit-rate and operating powers. Recently we reported in [6] some design rules to address this apparent deficiency within the literature. However, these rules were derived for idealized lossless fibers, which although adequate when using short lengths of low-loss nonlinear fiber, do not apply to the general case where the fiber losses are higher. The purpose of the current study is to generalize the scaling rules to include the case where the fiber loss is also included and then to apply and experimentally verify the validity of our design laws in a $2 \mathrm{R}$ set-up based on a $1 \mathrm{~km}$ length of HNLF.

\section{Scaling rules for lossy waveguide}

In our study we have adopted the same approach as in [6] by addressing the regenerator performance in relation to its transfer function (TF - output pulse energy vs. input pulse peak power). We mainly focus on the ability of the regenerator to: (i) improve the output extinction ratio between the ' 1 ' and ' 0 ' levels, and (ii) achieve amplitude equalization over a fixed input power range. We have modeled the nonlinear Schrödinger equation (NLSE) [7], and parameterized it in terms of normalized quantities such as the dispersion length $L_{D}=T_{0}^{2} /\left|\beta_{2}\right|$, the nonlinear length $L_{N L}=1 /\left(Y_{p}\right)$, the effective length $L_{e f f}=\left(1-e^{-\alpha L}\right) / \alpha$ and the 'soliton' number $N^{2}=L_{D} / L_{N L}$ (here $T_{0}$ and $P_{p}$ are respectively the 1/e half-width and the peak power of the input Gaussian pulse). The physical parameters of the fiber (second order dispersion $\beta_{2}$, nonlinearity coefficient $\gamma$, attenuation losses $\alpha$ and length $L$ ) can then be associated directly with normalized quantities, allowing us to drastically decrease the complexity of the regenerator design.

For a normal dispersion fiber $\left(\beta_{2}>0\right)$, our calculations demonstrate that, depending on the system parameters, the TF can either exhibit a local maximum (at the nominal operation point $P_{l}^{i n}$ ), or increase monotonously. At the boundary of these two operating regimes, the TF exhibits an extended locally flat region at $P_{l}^{\text {in }}$, around which the output pulse energy remains constant. This condition represents the optimum operational regime, and can be achieved for several combinations of parameter settings. We found the general condition that needs to be satisfied however can be described by the expression:

$$
\frac{L_{e f f} \cdot L}{L_{D}{ }^{2}} \approx \frac{N_{0}\left(\alpha L_{D}\right)}{N_{l}^{i n}}=\frac{N_{0}\left(\alpha L_{D}\right)}{\sqrt{L_{D} \gamma P_{l}^{i n}}}
$$

where, $N_{0}\left(\alpha L_{D}\right)$ is a parameter that is a weak function of $\alpha L_{D}$, and is found to be constant $(\sim 0.412)$ for $\alpha L_{D}$ values ranging from 0 up to $20 \mathrm{~dB}$ and thus still holds for the lossless case. Note that we derived a value of $\mathrm{N}_{0}=0.382$ in 
reference [6] and we attribute the discrepancy $(\sim 8 \%)$ between these values to be due to numerical round-off errors associated with fitting Eq. (1) over the large parameter space considered here.

For any given value of $\alpha L_{D}$, we can plot a multi-contour map that links the regenerator performance to the fiber parameters, the filter offset, and the required input peak power $P_{1}^{\text {in }}$ (as in the example shown in Fig.1c). Within this plot we can identify the three regimes of operation defined previously and as described in the associated caption (with the definition of the compression ratio $\rho$ from Fig.1b).

\section{Experimental validation}

We studied the validity of our design rules using the set-up shown in Fig.1a. A chirp compensated gain-switched DFB laser was used to generate a $10 \mathrm{GHz}$ pulse stream of nearly transform limited $\sim 6 \mathrm{ps}$ Gaussian pulses. The pulses were amplified to an average power of up to $30 \mathrm{dBm}$ in an erbium-doped amplifier and fed into a 1-km long HNLF. The HNLF exhibits a chromatic dispersion of $-1.7 \mathrm{ps} / \mathrm{nm} / \mathrm{km}$ at $1550 \mathrm{~nm}$, a dispersion slope of $0.023 \mathrm{ps} / \mathrm{nm}^{2} / \mathrm{km}$ and an attenuation loss of $2.13 \mathrm{~dB} / \mathrm{km}$. The nonlinearity coefficient of the fiber was $18 \mathrm{~W}^{-1} \cdot \mathrm{km}^{-1}$. At the output of the fiber, a tunable filter $(0.57 \mathrm{~nm}$ bandwidth) was used to filter the spectrally broadened spectrum. The exact pulse width was measured as $6.15 \mathrm{ps}+/-0.1 \mathrm{ps}$ (see Fig.3a), leading to a $L_{e f f} L / L_{D}{ }^{2}$ ratio ranging between 0.021 and 0.024 . Using these parameters and following the approach we described in the previous section, we modeled the performance of the regenerator for various values of the filter offset (Fig.1c). According to Eq.(1), optimum operation in terms of the TF characteristics can be achieved for a nominal peak power of $3.1 \mathrm{~W}(+/-0.3 \mathrm{~W})$. Fig.1c shows that this value can be achieved for a filter offset of $\sim 3.0 \mathrm{~nm}$.

(a)

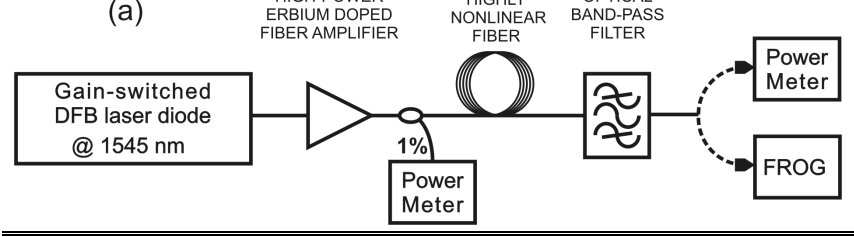

(b)

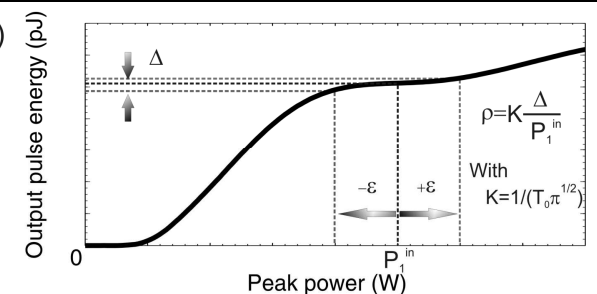

(c)

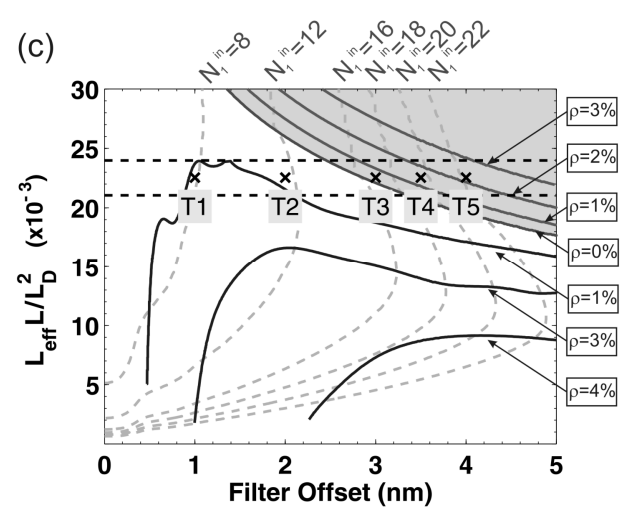

Fig. 1: (a) Experimental setup used for the characterization of the regenerator. (b) Definition of the compression ratio $\rho$ used to assess the transfer function regime at nominal input peak power $\mathrm{P}_{1}{ }^{\text {in }}$ for a tolerable power variation range of $\varepsilon=7.5 \%$. (Gaussian pulses are assumed at the output). (c) Contour map corresponding to the fiber used with iso-contour plots for several $\mathrm{N}_{1}{ }^{\mathrm{in}}$ and $\rho$ values. The shaded area corresponds to the region providing a monotonic transfer function, the line $\rho=0$ defines the optimum operational point defined by Eq. 1. The TF has a local maximum in the un-shaded region. The positions of the experimental points (crosses) are shown with corresponding upper and lower bound due to the uncertainty in the pulse width estimation (dashed lines).

We then measured several TFs for different output filter positions in order to demonstrate the existence of the different operation regimes. Fig. 2 shows TFs obtained when varying the filter position from $-1 \mathrm{~nm}$ to $-4 \mathrm{~nm}$. The plots show clearly the transition between the three operational regimes as the filter offset is varied, as predicted within Fig.1c. The plots in Fig. 2 also contain the corresponding modeled TFs (including the contribution of the dispersion slope and the extra insertion losses present in our system), highlighting the very good agreement between experimental and simulation results, especially for the range of input powers of practical interest. We attribute the discrepancies at higher input peak power levels to be due to differences in the detailed evolution of SPM generated spectral side lobes associated with residual chirp on the initial pulses.

In order to ensure that the pulse properties were preserved during the regeneration process, we next characterized the pulses in terms of both phase and intensity using Second Harmonic Generation Frequency Resolved Optical Gating (SHG-FROG) [8]. The measurements shown in Fig.3 were made for operation in the optimal regime. Fig.3b indicates that the output pulses remain close to transform-limited with the same slight amount of frequency chirp across the entire pulse, as reflected by the corresponding time-bandwidth product (TBP) values (Fig.3c). However, it should be noted that some of the shaping and chirp originates due to the output filter phase response itself. Some 
temporal pulse width variation is experienced at the level of $+/-5 \%$ around 5.8 ps. Although these fluctuations can be predicted, these variations are reflective of the fact that the regeneration process is defined by the overall pulse properties (intensity profile and peak power), and not just the local instantaneous phase shift as in an interferometric switch [3].
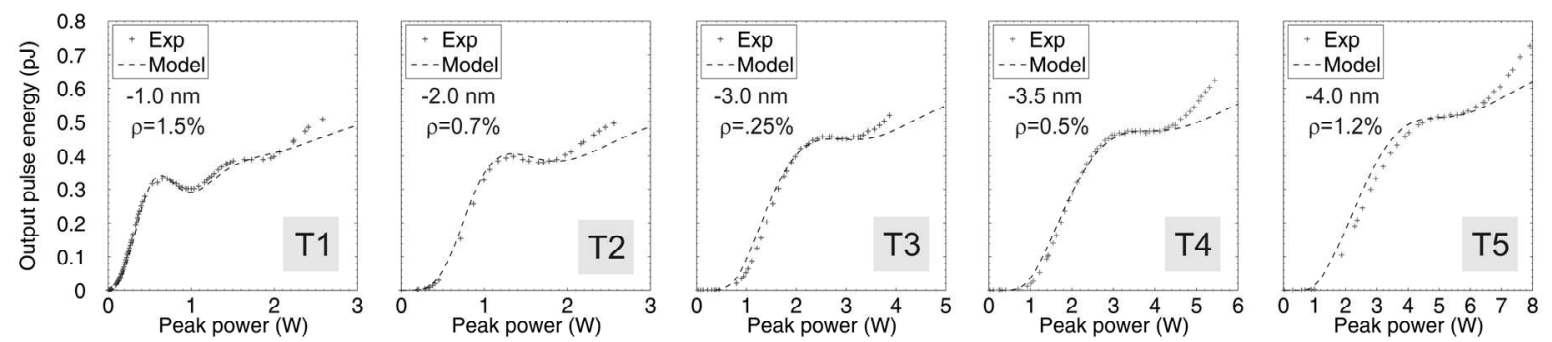

Fig. 2: Experimental and modeled transfer functions for five filter positions $(-1 \mathrm{~nm},-2 \mathrm{~nm},-3 \mathrm{~nm},-3.5 \mathrm{~nm}$, and $-4 \mathrm{~nm})$. Estimated compression ratio $\rho$ for experimental data is also reported.
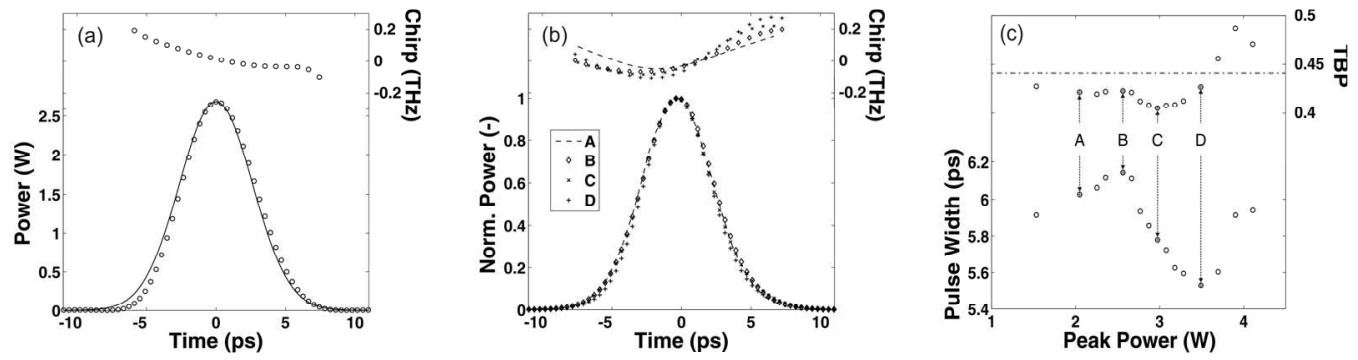

Fig. 3: (a) Temporal chirp (top) and intensity profile (bottom) of the pulses at the fiber input for an input peak power of $2.6 \mathrm{~W}$. The solid line corresponds to a Gaussian pulse of 6.15 ps width. (b) Temporal chirp and intensity profiles of the pulses after the output filter for four positions A,B C, and D corresponding to input peak powers of 2, 2.5, 3, and 3.5 W for case T3. (c) Corresponding retrieved pulse widths (bottom) and time-bandwidth products (top) from the FROG measurements corresponding to the $\mathrm{T} 3$ filter position.

\section{Conclusion}

We have extended the design of Self-Phase Modulation optical 2R regenerators to include the effects of fiber attenuation, and have experimentally confirmed the validity of our scaling rules. We have additionally performed detailed measurements of the output pulse characteristics, demonstrating the good reshaping capability of this form of regenerator when operating in the optimal regime. We believe that this work may lay the foundations for a pertinent cross-comparison of the performance of silica and compound glass HNLFs which have significantly different properties and which have both demonstrated good regeneration performance.

\section{Acknowledgements}

Christophe Finot gratefully acknowledges the financial support of the European Union through a Marie Curie grant. We gratefully acknowledge Furukawa Electric for providing the HNLF used in this experiment. This work was funded in part by the European Union Specific Targeted Research Project TRIUMPH IST-027638.

\section{References}

[1] P. V. Mamyshev, "All-optical data regeneration based on self-phase modulation effect," in Proc. European Conference on Optical Communications (ECOC'98) (1998), p. 475.

[2] P. Petropoulos, T. M. Monro, W. Belardi, K. Furusawa, J. H. Lee, and D. J. Richardson, "2R-regenerative all-optical switch based on a highly nonlinear fiber," Opt. Lett. 26, 1233-1235 (2001).

[3] M. Rochette, L. B. Fu, V. Ta'Eed, D. J. Moss, and B. J. Eggleton, "2R Optical Regeneration: An All-Optical Solution for BER Improvement," J. of Selected Topics in Quantum Electronics 12, 736-744 (2006).

[4] S. Asimakis, F. Parmigiani, P. Petropoulos, D. J. Richardson, N. Sugimoto, and F. Koizumi, "A 2-m-long Reshaping Regenerator Based on a Highly Nonlinear Bismuth Oxide Fiber," in Optical Fiber Communication Conference (OFC) 2006 (2006), p. OThB5.

[5] T.-H. Her, G. Raybon, and C. Headley, "Optimization of pulse regeneration at $40 \mathrm{~Gb} / \mathrm{s}$ based on spectral filtering of self-phase modulation in fiber," Photon. Technol. Lett. 16, 200-202 (2004).

[6] L. Provost, C. Finot, P. Petropoulos, and D. J. Richardson, "Design Scaling Laws for Self-Phase Modulation-based 2R-Regenerators," in Proc. European Conference on Optical Communications (ECOC'06) (2006), p. We 4.3.2.

[7] G. P. Agrawal, Nonlinear Fiber Optics, 3rd Edition (Academic Press, 2001).

[8] R. Trebino, Frequency-Resolved Optical Gating : the measurement of ultrashort laser pulses (Kluwer Academic Publishers, Norwell, MA 2000). 